\title{
RELAÇÃO ENTRE CAPACIDADES MOTORAS DE IDOSAS PRATICANTES DE HIDROGINÁSTICA E ALONGAMENTO
}

Marcelo Gava Pompermayer ${ }^{1}$ Andréa Kruger Gonçalves²

resumo

O objetivo do estudo foi verificar se há relação entre capacidades motoras de idosas submetidas a um programa físico composto por dois tipos de atividade física (hidroginástica e alongamento). A amostra foi composta por 23 indivíduos saudáveis e ativos do sexo feminino, com idades entre 60 e 80 anos. Os sujeitos foram divididos em dois grupos etários (GR1 = 60 a 69 anos, GR2 = 70 a 80). Os instrumentos foram testes físicos da bateria 'Senior Fitness Test' de Rikli e Jones (2008). As capacidades motoras avaliadas foram: flexibilidade de membros inferiores (FLEXMI); força de membros inferiores (FMI); flexibilidade de membros superiores (FLEXMS);

1 Graduando em Educação Física da Universidade Federal do Rio Grande do Sul (UFRGS). Bolsista do Programa de Educação Tutorial (PET). E-mail: marcelog.p@hotmail.com.

2 Doutora em Psicologia Social pela Universidade de São Paulo (USP). Professora do curso de Educação Física da Universidade Federal do Rio Grande do Sul (UFRGS). E-mail: andreakg@terra.com.br. 
força de membros superiores (FMS); agilidade/equilíbrio (AE); e resistência aeróbica (RESAER). O tratamento estatístico foi realizado através da correlação de Pearson do programa SPSS 18.0. Foram encontradas, no GR1, correlações entre: FMl e FLEXMl, FMl e AE, FMl e RESAER, FLEXMl e RESAER, AE e RESAER. No GR2, a única correlação encontrada foi entre AE e RESAER. Os componentes da aptidão física, nos idosos mais jovens, apresentaram, neste estudo, maior número de correlações, indicando que incrementos em uma capacidade motora podem gerar melhoras noutra. Estudos com diferentes atividades físicas são necessários para confirmar essas relações.

palavras - chave

Aptidão física. Envelhecimento. Hidroginástica. Alongamento.

Uma característica marcante dos países desenvolvidos e em desenvolvimento é o aumento da população idosa. O Brasil, em 2025, terá 34 milhões de pessoas acima de 60 anos, o que representa $15 \%$ da população total (GONÇALVES, 2001). A expectativa de vida aumenta significativamente e, com isso, a preocupação com a qualidade de vida para com esses indivíduos se torna mais evidente. Em relação ao envelhecimento, investigações sobre atividades físicas e funcionalidade dos idosos têm sido desenvolvidas.

Os aspectos físicos são um dos principais indicadores do processo de envelhecimento, porque, com o aumento da idade, manifestam-se alterações em diversos parâmetros fisiológicos e capacidades físicas. A função cardiorrespiratória perde eficiência, afetando negativamente o desempenho aeróbi$\mathrm{co}$, visto que o consumo máximo de oxigênio ( $\mathrm{VO}^{2}$ máx.) e a frequência cardíaca máxima diminuem. A capacidade de redirecionamento do sangue para os músculos ativos e a utilização de oxigênio pelos mesmos também sofre decréscimo durante o exercício, interferindo na performance. O desempenho anaeróbico diminui devido à perda de massa dos grandes grupos musculares, decréscimo no tamanho e no número de fibras musculares glicolíticas de contração rápida, menor fluxo sanguíneo intramuscular, contribuindo para uma remoção mais lenta do lactato sanguíneo (SPIRDUSO, 2006). 
De acordo com Pu e Nelson (2001), após a terceira década de vida, ocorre uma redução de $10 \%$, por década, da capacidade máxima de oxigênio em indivíduos sedentários saudáveis. Matsudo (2004) afirma que ocorre um declínio na força de $10 \%$ a $15 \%$, por década, podendo atingir $30 \%$, após os 70 anos. As mudanças anteriormente mencionadas influenciam as capacidades físicas, sendo que as relacionadas diretamente com a saúde são: resistência cardiorrespiratória, composição corporal e aptidão músculo-esquelética (força muscular, resistência muscular e flexibilidade) (NIEMAN, 1999). As capacidades físicas compõem a aptidão física e possibilitam a realização de atividades diárias, bem como a minimização do risco de doenças crônicas.

A flexibilidade apresenta decréscimos que se relacionam diretamente com a capacidade funcional do idoso, pois quanto maior a amplitude de movimento, menor o comprometimento da integridade músculo-tendíneo e articular (ALTER apud GERALDES et al., 2008)³. Declínios na flexibilidade são observados em razão, principalmente, à deficiência de colágeno que torna os tendões, ligamentos e cápsulas articulares mais rígidos (ADAMS, O'SHEA; O'SHEA apud AGUIAR; GURGEL, 2009)4. Essa diminuição está associada às dificuldades no desempenho de tarefas cotidianas, como utilizar transportes públicos, transpor desníveis no solo, ou caminhar (GUIMARÃES; FARINATTI, 2005), comprometendo a independência do idoso.

Para Geraldes et al. (2008), existe associação entre nível de atividade física e flexibilidade, sendo que idosos ativos, em comparação a idosos sedentários institucionalizados, apresentam maior autonomia, maiores níveis de flexibilidade geral, maior velocidade de caminhada, melhor aptidão cardiorrespiratória e melhor qualidade de vida. Morey et al. (apud UENO et al., $2000)^{5}$ apresenta um estudo longitudinal com idosos submetidos a um treino de 12 semanas, com três aulas semanais de exercícios aeróbicos, de força e de flexibilidade, onde houve melhora significativa da flexibilidade do quadril. Benedetti e Petroski (1999) indicam amplitudes da abdução de ombro necessárias para realizar determinadas atividades diárias: para tomar banho são necessários ângulos de até $170^{\circ}$; para lavar as costas: $130^{\circ}$; vestir calças: $60^{\circ}$; e o uso do toalete: $40^{\circ}$. Esses dados reforçam a importância de um treino de

\footnotetext{
3 ALTER, Michael J. Science of flexibility. Champaign: Human Kinetics, 1996. Apud GERALDES et al, 2008.

4 ADAMS, Kent; O'SHEA, Patrick; O'SHEA, Katie L. Aging: its effects on strength, power, flexibility, and bone density. Journal of Strength and Conditioning Research, Champaign, v. 21, n. 2, p. 65-77, 1999. Apud AGUIAR \& GURGEL, 2009.

5 MOREY, Miriam C. et al. Evaluation of a supervised exercise program in a geriatric population. Joumal of the American Geriatric Society, v. 37 n. 4, p. 348-354, 1989. Apud UENO et al. , 2000
} 
flexibilidade, não só na amplitude de movimento, mas também na autonomia, na funcionalidade, na independência e na qualidade de vida do idoso.

A resistência aeróbica é outra capacidade física considerada fundamental na rotina de exercícios para a população idosa. $\mathrm{O}$ treinamento aeróbico promove uma diminuição dos fatores de risco para doenças cardiovasculares e metabólicas, como observado no estudo de Monteiro et al. (2010), a partir de um treinamento aeróbico para idosas com diabetes tipo 2. Os resultados mostraram redução significativa da glicemia e da pressão arterial diastólica. Miranda e Rabelo (2006) avaliaram a capacidade aeróbica de idosas através do teste "andar 6 minutos" de Rikli e Jones após submetê-las a um programa de treinamento de aproximadamente três meses que consistia em aulas de dança, ginástica localizada, ginástica aeróbica, alongamento e atividades lúdicas. Os resultados, quando comparados aos valores padrões de referência da bateria de testes de Rikli e Jones, não apresentaram diferença estatisticamente significativa, indicando valores dentro da média referencial, e, portanto, um nível desejado de capacidade aeróbica para uma vida funcionalmente independente.

Exercícios de força são importantes, dentre outros aspectos, para a manutenção e aumento da massa muscular, que, durante o envelhecimento, sofre reduções devido ao processo de sarcopenia. A perda dessa massa magra implica na diminuição da resistência e da potência muscular, comprometendo a independência funcional do idoso.

No estudo de Trancoso e Farinatti (2002), com mulheres idosas submetidas a um treinamento com pesos, a mensuração da força muscular indicou aumento de $60 \%$ para o exercício "leg-press" e $62 \%$ para o supino reto. Há estudos com aumentos ainda mais expressivos na força muscular de idosos. Frontera et al. (1990) encontraram melhora de 100\% de 1 RM (repetição máxima) na força de extensores de joelho em homens idosos com idades entre 60 e 72 anos, após um treinamento de 12 semanas.

Os estudos supracitados demonstram a importância de um treinamento adequado para a população idosa, visando atingir todas as capacidades físicas; as adaptações decorrentes são importantes na promoção e manutenção da qualidade de vida dessa população. Diferentes modalidades de atividades físicas são escolhidas pelo público idoso, sendo que algumas são mais indicadas e preferidas. Entre elas, pode destacar-se a hidroginástica, a qual proporciona um ambiente favorável para a realização de exercícios físicos, em razão das propriedades físicas da água que levam a um melhor aproveitamento da atividade, oferecendo menores riscos à saúde (ALVES et al., 2004). 
A hidroginástica tradicional aproveita a resistência imposta pela água como sobrecarga, promove benefícios no condicionamento aeróbico, força muscular e composição corporal; quanto à flexibilidade, existem estudos controversos (PASSOS et al., 2008).

O alongamento é um dos métodos de trabalho da capacidade física flexibilidade, sendo indispensável para que o idoso obtenha uma melhor qualidade de vida e aptidão física. A flexibilidade pode ser trabalhada de diversas maneiras e através de diferentes atividades, sendo um dos principais meios, a realização de exercícios de alongamento estático, ativo ou passivo, durante um período de tempo que varia entre 15 e 60 segundos, aproximadamente.

$\mathrm{O}$ intuito de realizar este estudo foi verificar se existe relação entre as capacidades motoras de idosos submetidos a um programa físico composto por dois tipos de modalidades (hidroginástica e alongamento), partindo-se do pressuposto de que ao trabalhar com duas atividades distintas, estaria se estimulando diferentes capacidades físicas, as quais poderiam ter seus resultados potencializados. A hidroginástica é um tipo de atividade física que trabalha predominantemente a capacidade aeróbica, mas que abrange, também, o treino da força. Já na modalidade de alongamento, predomina o treinamento da flexibilidade, a qual seria a capacidade física não enfatizada na modalidade hidroginástica.

\section{Metodologia}

O tipo de estudo foi descritivo ex-pos-facto. A amostra foi composta por 23 indivíduos saudáveis e ativos do sexo feminino, com idades entre 60 e 80 anos e participantes do projeto de extensão do Centro de Esporte, Lazer e Recreação do Idoso (CELARI), da Universidade Federal do Rio Grande do Sul (UFRGS). As idosas foram participantes de oficinas de hidroginástica e alongamento, com quatro aulas semanais (duas de cada atividade) de $45 \mathrm{mi}$ nutos cada, durante cinco meses. Fizeram parte da amostra, todas as idosas que participaram da avaliação e que frequentaram as aulas durante o período do estudo. Os sujeitos foram, ainda, divididos em dois grupos etários: grupo 1 (GR1) entre 60 e 69 anos, grupo 2 (GR2) entre 70 a 80 anos.

A oficina de alongamento era subdividida em: parte inicial (10-12 minutos), que continha exercícios de mobilização articular e de ativação cardiocirculatória aeróbia, com o intuito de aumentar o fluxo sanguíneo e diminuir a rigidez muscular e articular; a parte principal (30 minutos) consistia em alongamentos estáticos passivos, em sua maioria, com duração média de 
30 segundos cada, visando os principais grupos musculares (posteriores de coxa e perna, anteriores de coxa e pelve, peitorais, costas, etc.), inicialmente com os alunos em pé, posteriormente sentados e, em seguida, deitados; a sessão tinha fim com um breve relaxamento (5 minutos).

A hidroginástica subdividia-se em: parte inicial (7-10 minutos), que consistia em mobilização articular e aquecimento geral para preparar o corpo para o exercício. A parte principal foi separada em resistência aeróbica e resistência muscular localizada. A resistência aeróbica durava de 20 a 25 minutos e caracterizava-se por exercícios que movimentassem simultaneamente membros superiores e inferiores. A intensidade dos exercícios aeróbicos foi controlada pela percepção subjetiva de esforço através da Escala de Borg. A resistência muscular localizada trabalhava separadamente os segmentos e grupos musculares e durava aproximadamente 10 minutos; foi usado para esses exercícios o auxílio da barra fixada nas bordas da piscina e aqua-tubos. $\mathrm{Na}$ parte final, era realizado um alongamento geral (5 minutos).

O protocolo usado para mensuração da aptidão física dos sujeitos foi o de Rikli e Jones (2008), com avaliação da força de membros inferiores (FMI), força de membros superiores (FMS), flexibilidade de membros inferiores (FLEXMI), flexibilidade de membros superiores (FLEXMS), agilidade/equilíbrio (AE) e resistência aeróbica (RESAER). Os testes da bateria foram: a) FMI - levantar e sentar; b) FMS - flexão de antebraço; c) FLEXMI - sentado e alcançar; d) FLEXMS - alcançar atrás das costas; e) AE - sentado, caminhar 2,44m e voltar a sentar; f) RESAER - andar 6 minutos.

A análise estatística foi realizada através de correlação de Pearson no programa SPSS 18.0 com nível de significância $\mathrm{p}<0,05$.

\section{Resultados e Discussão}

As correlações foram significativas de modo mais acentuado no GR1. A FMI apresentou correlação significativa com a FLEXMI, AE e RESAER. A FLEXMI se correlacionou significativamente com FMI e RESAER. A AE demonstrou significância com FMI e RESAER. A RESAER correlacionou-se com FMI, FLEXMI e AE. Em relação à AE é importante destacar que as correlações foram sempre negativas, isto é, quanto menor o tempo para realização do teste de AE (o que indica melhores resultados), maior as outras capacidades correlacionadas. No GR 2, houve apenas correlação significativa da AE com a RESAER, sendo negativa. 
A correlação encontrada na FMI, com a FLEXMI, pode estar relacionada com o aumento de sarcômeros em série. O sarcômero é a unidade funcional básica da miofibrila e a unidade de contração muscular básica (WILMORE; COSTILL; KENNEY, 2010). Aumentos na amplitude de movimento podem ser causados por mudanças no comprimento muscular, sendo resultado da adição de sarcômeros em série. Essa adaptação envolve os filamentos de actina e miosina, possibilitando ao músculo gerar maiores níveis de força em seu novo comprimento funcional (WILLIAMS; GOLDSPINK, 1978). Outra explicação, é que trabalhos que estimulem a força (no caso, a hidroginástica) levam a um aumento na amplitude de movimento devido à própria execução dos exercícios, pois há alterações nas propriedades viscoelásticas (o que possibilita maior armazenamento de energia potencial nos componentes elásticos dos músculos, devido a um tecido menos rígido) melhorando o mecanismo do ciclo alongamento-encurtamento (BARAK; AYALON; DVIR apud VALE et al., 2006) ${ }^{6}$. Dessa forma, o trabalho conjunto de alongamento e hidroginástica, parece contribuir para o aumento da força e da flexibilidade, através da adição de sarcômeros em série e alterações nas propriedades viscoelásticas.

A correlação da FMI com a AE é importante, pois a agilidade e o equilíbrio são imprescindíveis para que o idoso se mantenha ativo e funcional. Carvalho e Soares (2004), em seu estudo de revisão, relataram que a potência muscular e a força de membros inferiores podem contribuir significativamente para a redução do risco de quedas em idosos. Alves et al. (2004) avaliaram os componentes da aptidão física (através do protocolo de Rikli e Jones) de 30 mulheres (o estudo contou também com um grupo controle de 30 mulheres) que praticaram hidroginástica duas vezes por semana, durante um período de três meses. O pós-teste dos praticantes indicou aumento na força de membros inferiores, quando comparados ao pré-teste do mesmo grupo e também quando comparados ao pós-teste do grupo controle. Cardoso, Mazo e Balbé (2010) também encontraram incrementos significativos na força de membros inferiores de 21 mulheres idosas submetidas a um trabalho de hidroginástica, com frequência de duas vezes por semana, durante 72 semanas. Resende, Rassi e Vianna (2008) realizaram um estudo com 25 idosas que praticaram hidroterapia por 12 semanas; analisando o equilíbrio, observaram aumentos significativos. Os autores citam outros estudos em que se observam melhoras no equilíbrio associado a um menor risco de quedas.

6 BARAK, Yaron; AYALON, Moshe; DVIR, Zeevi. Transferability of Strength Gains from Limited to Full Range of Motion. Medicine and Science in Sports and Exercise, v. 36, n. 8, p. 1413-1420, 2004. Apud VALE et al., 2006. 
A queda no idoso é muito recorrente, e esse fato pode levar ao isolamento por parte do próprio indivíduo e receio de realizar atividades diárias, aumentando a predisposição aos problemas de saúde. Aproximadamente $30 \%$ dos idosos sofrem, ao menos, uma queda por ano (GUIMARÃES; FARINATTI, 2005). Esse número é preocupante, porque caso ocorra uma fratura, será necessária imobilização e, se o período for prolongado, há chances de ocorrência de atrofia muscular grave, pois o desuso causa mais perdas nas idades avançadas. A força de membros inferiores garante ao idoso uma base de sustentação e possibilita nas situações de instabilidade, um restabelecimento breve do equilíbrio.

As correlações encontradas com a RESAER (tanto em relação à FLEXMI, FMI quanto à AE) parecem estar mais relacionadas com a menor faixa etária do GR1, visto que no GR2 encontrou-se correlação apenas com RESAER. A resistência aeróbica sofre decréscimos relacionados à idade, a frequência cardíaca máxima diminui, a capacidade de redirecionamento de sangue para os tecidos ativos é menor e assim o $\mathrm{VO}^{2}$ max também diminui (SPIRDUSO, 2006).

No GR 2, a AE se correlacionou significativamente, mas de maneira inversa, com a RESAER, ou seja, quanto menor o tempo para realizar o teste de agilidade/equilíbrio, maior distância foi percorrida no teste aeróbico. Fato ocorrido também no GR1 com AE. No teste de AE, as fibras mais recrutadas são as de contração rápida, pois é um teste intenso e de curta duração. No teste de RESAER, por outro lado, as fibras de contração lenta são mais recrutadas. Não foram encontrados, na literatura, estudos para justificar tal correlação, indicando a necessidade de pesquisas focadas nessa relação.

Em relação à FMS e à FLEXMS não foram encontradas, em nenhum dos grupos, correlações significativas. A força e a flexibilidade dos membros inferiores se correlacionaram, como foi exposto anteriormente, devido, possivelmente, à adição de sarcômeros em série e alterações nas propriedades viscoelásticas; a falta de resultados acerca dos membros superiores sugere que estes mecanismos não ocorreram de maneira significativa, indicando a necessidade de reavaliação dos exercícios para membros superiores das aulas de alongamento e hidroginástica.

\section{Conclusão}

A prática da hidroginástica e do alongamento demonstrou, neste estudo, que há correlações entre: a força dos membros inferiores (FMI) e a 
flexibilidade dos membros inferiores (FLEXMI); a força de membros inferiores (FMI) e agilidade/equilíbrio (AE); a força de membros inferiores (FMI) e a resistência aeróbica (RESAER); a flexibilidade de membros inferiores (FLEXMI) e a resistência aeróbica (RESAER); a agilidade/equilíbrio (AE) e a resistência aeróbica (RESAER). Entretanto, não foi encontrada nenhuma correlação envolvendo a flexibilidade de membros superiores (FLEXMS) e a força de membros superiores (FMS).

O teste de correlação de Pearson indicou maior número de correlações significativas no grupo idoso de menor idade (GR1), indicando que melhoras em uma capacidade motora pode gerar incrementos noutra.

Pode-se, então, potencializar os resultados físicos no sentido de que, treinando um aluno numa modalidade específica de flexibilidade de membros inferiores, por exemplo, ele pode obter ganhos também na força de membros inferiores. Dessa forma, levando em conta as possíveis relações entre as capacidades físicas, o aluno pode fazer atividades de sua preferência, mesmo que inicialmente esse não seja o objetivo da modalidade em questão. Exemplificando, se determinado aluno não aprecia musculação, mas precisa aumentar a força de membros inferiores, podem ser indicadas aulas de alongamento, mesmo que o objetivo da aula seja aumento da flexibilidade. Essa estratégia pode melhorar o engajamento e a adesão de idosos às atividades físicas regulares. Porém, essas são questões que necessitam de outras pesquisas mais específicas para averiguação.

\section{RELATIONSHIP BETWEEN MOTOR PERFOR- MANCE IN ELDERLY PRACTICED HYDROGYM- NASTICS AND STRETCHING}

\section{abstract}

The aim of the study was to verify if there is association between the motor capacities of older women undergoing a physical program composed of two types of activities (hydrogymnastics and stretching). The sample consisted of 23 healthy and active female, aged between 60 and 80 years. The subjects were divided into two age groups (GR1 $=60$ to 69 years, GR2 $=70$ to 80 ). The instrument was the 'Senior Fitness Test' of Rikli and Jones (2008). Motor capacities evaluated were: lower limbs flexibility (FLEXMI), lower limbs strength (FMI), upper limbs flexibility (FLEXMS), upper limbs strength (FMS), 
agility / balance (AE) and aerobic resistance (RESAER). Statistical analysis was performed using the Pearson's Correlation of SPSS 18.0. In GR1, the correlations were found between: FMl e FLEXMI, FMl e AE, FMl e RESAER, FLEXMl e RESAER, AE e RESAER. In GR2, the only correlation was found between AE and RESAER. The components of physical aptitude, in younger elderly, presented, in this study, a higher number of correlations, indicating that increases in a component can lead to improvements in another. Studies with different types of activities are needed to confirm these relationships.

keywords

Physical aptitude. Aging. Hydrogymnastics. Stretching.

Tabela 1 - Coeficientes de correlação de Pearson de componentes da aptidão física de idosos divididos em dois grupos de idade: GR 1 (60-69 anos), GR 2 (70-80 anos)

\begin{tabular}{|c|c|c|c|c|c|c|c|c|}
\hline GR & $\begin{array}{l}\text { Capac. } \\
\text { motora }\end{array}$ & & FMl & FMS & FLEXMI & $A E$ & FLEXMS & REASER \\
\hline \multirow[t]{12}{*}{1,00} & FMl & $\mathrm{CCP}$ & 1 & ,465 &, $672\left(^{*}\right)$ &,$- 635\left(^{*}\right)$ & -,098 &, $697\left(^{\star}\right)$ \\
\hline & & Sig. & & , 175 & ,033 & ,049 & ,789 &, 025 \\
\hline & FMS & $\mathrm{CCP}$ & ,465 & 1 & ,409 &,- 454 &,- 064 &, 551 \\
\hline & & Sig. &, 175 & & ,241 & , 188 & 860 & ,099 \\
\hline & FLEXMI & $\mathrm{CCP}$ &, $672\left(^{\star}\right)$ & ,409 & 1 &,- 318 &,- 132 &, $672\left(^{*}\right)$ \\
\hline & & Sig. & ,033 & ,241 & & , 370 &, 716 & ,033 \\
\hline & $\mathrm{AE}$ & $\mathrm{CCP}$ &,$- 635\left(^{\star}\right)$ &,- 454 &,- 318 & 1 &,- 356 &,$- 684\left(^{*}\right)$ \\
\hline & & Sig. & ,049 & ,188 & ,370 & &, 313 & ,029 \\
\hline & FLEXMS & $\mathrm{CCP}$ &,- 098 &,- 064 &,- 132 &,- 356 & 1 & ,400 \\
\hline & & Sig. &, 789 & 860 &, 716 & ,313 & & ,252 \\
\hline & REASER & CCP &, $697\left(^{\star}\right)$ &, 551 &, $672\left(^{\star}\right)$ &,$- 684\left(^{*}\right)$ & ,400 & 1 \\
\hline & & Sig. & ,025 & ,099 & ,033 & ,029 & 252 & \\
\hline \multirow[t]{5}{*}{2,00} & FMl & CCP & 1 & ,396 &,- 196 &,- 374 &,- 253 & ,333 \\
\hline & & Sig. & &, 180 &, 522 & ,208 &, 404 &, 266 \\
\hline & FMS & $\mathrm{CCP}$ & ,396 & 1 &,- 146 &,- 211 &,- 547 & ,497 \\
\hline & & Sig. & , 180 & & ,634 & ,488 & ,053 & ,084 \\
\hline & FLEXMI & $\mathrm{CCP}$ &,- 196 &,- 146 & 1 &,- 091 & , 173 & ,125 \\
\hline
\end{tabular}


(continuação)

\begin{tabular}{cccccccc} 
& Sig. &, 522 &, 634 & &, 767 &, 572 &, 685 \\
AE & CCP &,- 374 &,- 211 &,- 091 & 1 &, 033 &,$- 752\left(^{\star \star}\right)$ \\
& Sig. &, 208 &, 488 &, 767 & &, 915 &, 003 \\
FLEXMS & CCP &,- 253 &,- 547 &, 173 &, 033 & 1 &,- 103 \\
& Sig. &, 404 &, 053 &, 572 &, 915 & &, 738 \\
REASER & CCP &, 333 &, 497 &, 125 &,$- 752\left({ }^{* \star}\right)$ &,- 103 & 1 \\
& Sig. &, 266 &, 084 &, 685 &, 003 &, 738 & \\
\hline
\end{tabular}

referências

AGUIAR, Jaina Bezerra; GURGEL, Luilma Albuquerque. Investigação dos efeitos da hidroginástica sobre a qualidade de vida, a força de membros inferiores e a flexibilidade de idosas: um estudo no Serviço Social do Comércio - Fortaleza. Revista Brasilleira de Educação Física e Esporte, São Paulo, v. 23, n. 4, p. 335-344, out./dez. 2009.

ALVES, Roseane Victor et al. Aptidão física relacionada à saúde de idosos. Revista Brasileira de Medicina do Esporte, São Paulo, v. 10, n. 1, p. 31-37, jan./fev. 2004.

BENEDETTI, Tânia Rosane Bertoldo; PETROSKI, Edio Luiz. Idosos asilados e a prática de atividade física. Revista Brasileira de Atividade Física e Saúde, Londrina, v. 4, n. 3, p. $5-16,1999$

CARDOSO, Adilson Sant'Ana; MAZO, Giovana Zarpellon; BALBÉ, Giovane Pereira. Níveis de força em mulheres idosas praticantes de hidroginástica: um estudo de dois anos. Revista Motriz, Rio Claro, v. 16, n. 1, p. 86-94, jan./mar. 2010.

CARVALHO, Joana; SOARES, José M. C. Envelhecimento e força muscular - breve revisão. Revista Portuguesa de Ciências do Desponto, Porto, v. 4, n. 3, p. 79-93, jul./ dez. 2004

FRONTERA, Walter R. et al. Strength training and determinants of $\mathrm{VO}^{2}$ max in older men. Joumal of Applied Physiology, v. 68, n. 1, p. 329-333, jan. 1990. [abstract]

GERALDES, Amandio Aristides Rihan et al. Correlação entre flexibilidade das articulações glenoumerais e coxofemorais e o desempenho funcional de idosas fisicamente ativas. Revista Brasileira de Fisioterapia, São Carlos, v. 12, n. 4, p. 274-82, jul./ago. 2008

GONÇALVES, Andréa Kruger. Novo ritmo na terceira idade. Revista Fundação de Amparo à Pesquisa do Estado de São Paulo - FAPESP, v. 8, n. 67, p. 01-03, ago. 2001.

GUIMARÃES, Joanna Miguez Nery; FARINATTI, Paulo de Tarso Veras. Análise descritiva de variáveis teoricamente associadas ao risco de quedas em mulheres idosas. Revista Brasileira de Medicina do Esporte, São Paulo, v. 11, n. 5, p. 299-305, set./out. 2005.

MATSUDO, Sandra Marcela Mahecha. Avaliação do Idoso - Física e Funcional. Londrina: Midiograf, 2004

MIRANDA, Érica Pinto; RABELO, Heloisa Thomaz. Efeitos de um programa de atividade física na capacidade aeróbia de mulheres idosas. Movimentum - Revista Digital de Edu cação Física, Ipatinga, v. 1, ago./dez., p. 1-13, 2006. 
MONTEIRO, Luciana Zaranza et al. Redução da pressão arterial, da IMC e da glicose após treinamento aeróbico em idosos com diabete tipo 2. Arquinos Brasilleiros de Cardiologia, São Paulo, v. 95, n. 5, p. 563-570, out. 2010.

NIEMAN, David C. Exercício e saúde. São Paulo: Manole, 1999.

PASSOS, Betânia Maria Araújo et al. Contribuições da hidroginástica nas atividades da vida diária e na flexibilidade de mulheres idosas. Revista da Educação Física, Maringá, v. 19, n. 1, p. 71-76, 2008.

PU, Charles T.; NELSON, Miriam E. Envelhecimento, função e exercício. In: FRONTERA, Walter R.; DAWSON, David. M.; SLOVIK, David. M. Exercício físico e reabilitação. Porto Alegre: Artmed, 2001. p. 347-372.

RESENDE, Selma Mendes; RASSI, Cláudia Maria; VIANA, Fabiana Pavan. Efeitos da hidroterapia na recuperação do equilíbrio e prevenção de quedas em idosas. Revista Brasileira de Fisioterapia, São Carlos, v. 12, n. 1, p. 57-63, jan./fev. 2008.

RIKLLI, Roberta E.; JONES, Jessie C. Teste de Aptidão Física para Idosos. Barueri - SP: Manole, 2008.

SPIRDUSO, Waneen W. Dimensões fisiológicas do envelhecimento. Barueri - SP: Manole, 2005.

TRANCOSO, Ericka Sant'Ana Federici; FARINATTI, Paulo de Tarso Veras. Efeitos de 12 semanas de treinamento com pesos sobre a força muscular de mulheres com mais de 60 anos de idade. Revista Paulista de Educação Física, São Paulo, v. 16, n. 2, p. 220-229, jul./dez. 2002.

UENO, Linda Massako et al. Análise dos efeitos quantitativos e qualitativos de um programa de educação física sobre a flexibilidade do quadril em indivíduos com mais de 60 anos. Revista Motriz, Rio Claro, v. 6, n. 1, p. 9-16, jun. 2000.

VALE, Rodrigo Gomes de Souza et al. Efeitos do treinamento resistido na força máxima, na flexibilidade e na autonomia funcional de mulheres idosas. Revista Brasileira de Cineantropometria e Desempenho Humano, Florianópolis, v. 8, n. 4, p. 52-58, 2006.

WILLIAMS, Pamela E.; GOLDSPINK, Geoffrey. Changes in sarcomere length and physiological properties in immobilized muscle. Joumal of Anatomy, v. 127, n. 3, p. 459-68, dec. 1978.

WILMORE, Jack H.; COSTILL, David L.; KENNEY, Larry W. Fisiologia do Esporte e do Exercício. Barueri - SP: Manole, 2010.

Recebido: 23/12/2010

1ª Revisão: 28/06/2011

$2^{a}$ Revisão: 30/09/2011

Aceite Final: 24/10/2011 